\title{
A 160-Gb/s OTDM Demultiplexer Based on Parametric Wavelength Exchange
}

\author{
Mengzhe Shen, Xing Xu, T. I. Yuk, and Kenneth Kin-Yip Wong, Member, IEEE
}

\begin{abstract}
Parametric wavelength exchange (PWE) has been demonstrated as a versatile device in providing different functionalities. In this paper, we will concentrate, numerically and experimentally, on one of these functionalities, namely, all-optical time demultiplexing of 160-Gb/s return-to-zero (RZ) signals based on a pulsed-pump PWE in a $400 \mathrm{~m}$ highly nonlinear dispersion-shifted fiber. Experimental results show power penalties $\leq 2.7 \mathrm{~dB}$ at bit-error rate of $10^{-9}$ for all demultiplexed $10-\mathrm{Gb} / \mathrm{s}$ $\overline{\mathbf{R} Z}$ signals. We also derive theoretical expressions for the conversion/residual efficiencies and investigate the impact of pump pulse width and phase mismatch on these efficiencies. Furthermore, the impacts of pulsed-pump wavelength and power level on the characteristics of the switching window are investigated numerically. As a result, the demultiplexer can be easily upgraded to an add-drop multiplexer because of the complete exchange nature of PWE, which is justified by the surviving channels' waveform performance.
\end{abstract}

Index Terms-Optical communication, optical signal processing, optical time-division multiplexing (OTDM), parametric wavelength exchange (PWE).

\section{INTRODUCTION}

$\mathbf{P}$ ARAMETRIC wavelength exchange (PWE) relies on four-wave mixing (FWM) phenomenon, where a signal wavelength at $\lambda_{\text {signal }}$ and an idler wavelength at $\lambda_{\text {idler }}$ exchange their power periodically while two strong pumps at $\lambda_{p 1}$ and $\lambda_{p 2}$ are copropagating in highly nonlinear dispersion-shifted fibers (HNL-DSF) [1]-[8]. According to the location of pumps in wavelength assignment, PWE can be classified into two categories, Type I (both pumps in normal dispersion region or denoted as WE I) and Type II (both pumps in anomalous dispersion region or denoted as WE II). Past results show that the pump-induced Raman amplification introduced asymmetric power transfer that degrades the performance of the WE I process [2]. Such performance degradation is particularly severe when the two pumps are arranged orthogonally in the normal dispersion region. Thus, WE II configuration with two pumps in the anomalous dispersion regime is presented [3]. In theory, no Raman gain is provided by pumps allocated in anomalous dispersion region. Attentive readers may wonder

Manuscript received March 14, 2009; revised May 29, 2009. Current version published September 30, 2009. This work was supported in part by grants from the Research Grants Council of the Hong Kong Special Administrative Region, China, under Grant HKU 7172/07E and Grant HKU 7179/08E.

The authors are with the Department of Electrical and Electronic Engineering, The University of Hong Kong, Hong Kong (e-mail: mzshen@eee.hku.hk; xuxing@eee.hku.hk; tiyuk@eee.hku.hk; kywong@eee.hku.hk).

Color versions of one or more of the figures in this paper are available online at http://ieeexplore.ieee.org.

Digital Object Identifier 10.1109/JQE.2009.2026653 whether the resultant Raman loss effect will have a detrimental effect in WE II. In experiment, if the signal power at fiber input is kept much lower than the threshold condition of Raman effects, it can safely predict that the Raman effects are insignificant. In fact, by using $P_{0}^{c r} \approx 16 A_{\text {eff }} / L_{\text {eff }} g_{R}$ [9], we can predict that the Raman threshold in practical WE II experiments. For HNL-DSF with $L_{\text {eff }}=1 \mathrm{~km}$ and $A_{\text {eff }}=7.5 \mu \mathrm{m}^{2}$, the predicted Raman threshold is $110 \mathrm{~mW}$. It means that Raman loss will not affect WE II performance as the typical input signal power is maintained at $3 \mathrm{dBm}$. We have also investigated the tuning range of WE II by fixing one pump wavelength and its corresponding signal wavelength, while tuning the other pump and its corresponding signal wavelength [4]. A tuning range of $15 \mathrm{~nm}$ is achieved with performance slightly degraded when one of the signals is tuned near the zero-dispersion wavelength (ZDW). The dynamic range of WE II was also explored recently [5], in which the crosstalk mechanism degrading the exchanged signal performance was discussed in detail. These discoveries support the practical use of PWE in modern optical network. For example, it may find applications as wavelength convertor or optical cross-connect switch in wavelength-division multiplexing (WDM) optical network [6]. Another interesting application of PWE is using it as optical packet switch [7] or demultiplexing in optical time-division-multiplexing (OTDM) systems [8]. In [7], an experimental validation of a PWE-based packet switch with two 10-Gb/s programmable nonreturn-to-zero (NRZ) signals that are switched in compliance with two synchronized quasi-square-pulsed pumps in the time domain was implemented.

The OTDM technology has advanced significantly over the past few years and extensive research efforts have been pursued in high-speed transmission systems over $160-\mathrm{Gb} / \mathrm{s}$ or higher (320 Gb/s and $640 \mathrm{~Gb} / \mathrm{s})$ [10]-[12]. For the practical implementation of optical packet switching, which brings the flexibility and efficiency of the Internet to transparent optical networking, one of the most essential devices is the high-speed channel demultiplexer. It selectively drops a $10-\mathrm{Gb} / \mathrm{s}$ or $40-\mathrm{Gb} / \mathrm{s}$ base rate tributary from a multiplexed high bit-rate data stream in the time domain. A switching window with high-extinction ratio is thus required to realize good drop performance.

Previously, channel demultiplexing has been realized by using FWM in conventional DSF with optical filtering [13] or cross-phase modulation in nonlinear optical loop mirror (NOLM) [14]. However, these approaches have their own merits and drawbacks. In general, the required length of conventional DSF is long, which will result in walk-off between data and gating pulses. Additionally, long fibers are less robust 


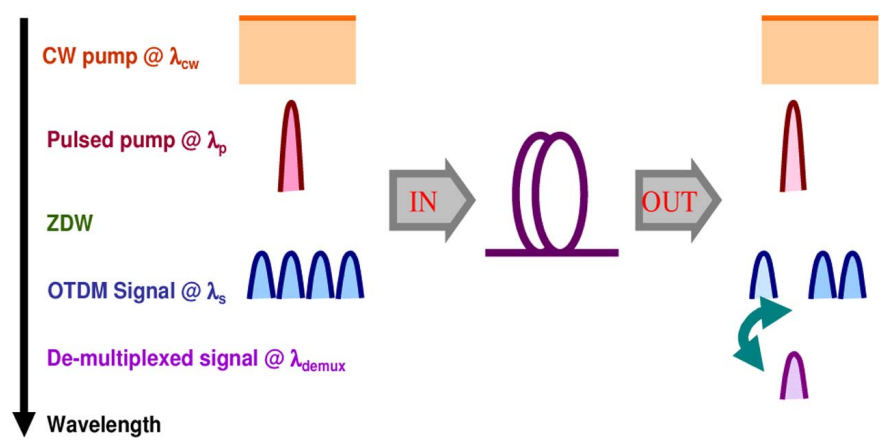

Fig. 1. Principle of operation of our proposed wavelength exchange-based demultiplexer.

to environmental disturbance and the polarization dependence is higher. Another design philosophy that aims at decreasing the size of the whole system is to replace fiber devices with semiconductor optical amplifier (SOA) [15]. The main limitation of an SOA-based approach results from its relatively slow temporal response governed by the carrier lifetime. Recently, pulsed-pump PWE has been studied, in which a synchronized pulsed pump and a continuous-wave (CW) pump were used in a PWE-based 80-Gb/s OTDM demultiplexer [16].

The operating principle of the proposed OTDM demultiplexer is shown in Fig. 1. A multiplexed input data pulse stream at a wavelength of $\lambda_{s}$ enters the fiber input whilst a synchronized control pulse at $\lambda_{p}$ and a CW pump at $\lambda_{\mathrm{cw}}$ are also fed into the fiber input as shown in Fig. 1. A selectively demultiplexed channel (Drop Channel) by the synchronized control pulse is produced at the wavelength of $\lambda_{\text {demux }}$, while the surviving channels (Through Channels) remain at the original wavelength. Due to the equipment constraint, the pulsed pump is prepared through wavelength conversion in a $100-\mathrm{m}$ dispersion-flattened photonic crystal fiber. The walk-off effect in the nonlinear fiber under a finite fiber birefringence results in a pulse pedestal. This walk-off effect is the major limitation for higher speed operations and can be overcome by using a separate pulse source to serve as the pulsed pump.

This paper is organized as follow. In Section II, we analyze the characteristics of switching window of our proposed demultiplexer both theoretically and numerically. In Section III, we demonstrate a $160-\mathrm{Gb} / \mathrm{s}$ signal demultiplexer using pulsedpump WE I in a 400-m-long HNL-DSF. We present the experimental results and discussions in Section IV. Our experiment is performed at a multiplexed data rate of $160 \mathrm{~Gb} / \mathrm{s}$ with a $10-\mathrm{Gb} / \mathrm{s}$ base rate and power penalty $\leq 2.7 \mathrm{~dB}$ at $10^{-9}$ is achieved for all demultiplexed $10-\mathrm{Gb} / \mathrm{s}$ return-to-zero (RZ) signals. The complete exchange nature between the signals enables the demultiplexer to be easily upgraded to high-speed add-drop multiplexer (ADM), which will be proved by clear surviving channels' waveforms. Finally, some concluding remarks are presented in Section V.

\section{TheORETICAL AND SimUlation Results}

PWE transfers optical power between signal and idler periodically [1], [2]. In an optical fiber, the process is most easily described by a set of coupled equations that describes the amplitudes of signal $A_{3}$ and idler $A_{4}$ as

$$
\begin{aligned}
\frac{\partial A_{3}}{\partial z}= & i \gamma\left[A_{3}^{2}+2 A_{1}^{2}+\frac{2}{3}\left(A_{2}^{2}+A_{4}^{2}\right)\right] A_{3} \\
& +\frac{2}{3} i \gamma A_{1} A_{2}^{*} A_{4} \exp (i \Delta \beta z) \\
& -\left(\beta_{13} \frac{\partial}{\partial t}-\frac{i}{2} \beta_{23} \frac{\partial^{2}}{\partial t^{2}}-\frac{\alpha}{2}\right) A_{3} \\
\frac{\partial A_{4}}{\partial z}= & i \gamma\left[A_{4}^{2}+2 A_{1}^{2}+\frac{2}{3}\left(A_{2}^{2}+A_{3}^{2}\right)\right] A_{4} \\
& +\frac{2}{3} i \gamma A_{1}^{*} A_{2} A_{3} \exp (i \Delta \beta z) \\
& -\left(\beta_{14} \frac{\partial}{\partial t}-\frac{i}{2} \beta_{24} \frac{\partial^{2}}{\partial t^{2}}-\frac{\alpha}{2}\right) A_{4} .
\end{aligned}
$$

Here $A_{j}$ is the slowly varying envelope of the optical field with frequency $\omega_{j}$, inverse group velocity $\beta_{1 j}$, and group-velocity dispersion (GVD) parameter $\beta_{2 j} . A_{1}, A_{2}, A_{3}$, and $A_{4}$ correspond to the amplitudes of two pumps, signal and idler, respectively. $z, \alpha$, and $\gamma$ represent the length of fiber transmission, the fiber loss and nonlinear coefficient, respectively. The propagation constant mismatch is defined as $\Delta \beta=\beta_{1}+\beta_{4}-\beta_{2}-\beta_{3}$, where $\beta_{j}$ are the propagation constants in the fiber. Please note that we do not include Raman gain coefficient between pumps and signal in (1). Thus, strictly speaking, it can be used to describe WE I only if Raman interaction is neglected. A theoretical expression for the shape of switching window based on similar set of equations has been obtained in [17] by assuming that the signal power levels to be unaffected by the nonlinearity in fiber optical parametric amplifier. Unfortunately, it does not apply to the context of PWE-based demultiplexing because the power at $A_{3}$ is transferring to $A_{4}$ during PWE process. An analytical solution involving elliptical functions for (1), which takes pump depletion into account, is discussed in [18], [19]. Nevertheless, it is not practical to obtain a time-dependant analytical solution by using it. Hence, we follow the method described in [20] to derive a time-dependant solution for (1). If we assume that the pumps are not depleted $\left(A_{1}, A_{2} \gg A_{3}\right)$ and neglect dispersion effect, the solutions for signal and idler are

$$
\begin{aligned}
& A_{3}(z)=\left(A_{3}(0) \cos g z-A_{3}(0) \frac{i \kappa}{2 g} \sin g z\right) \\
& A_{4}(z)=\left(i \frac{2}{3} \gamma A_{1}(0) A_{2}(0) A_{3}(0) \frac{\sin g z}{g}\right)
\end{aligned}
$$

where the parametric gain $g$ is defined as

$$
\begin{aligned}
g & =\sqrt{(k / 2)^{2}+4 \gamma^{2}\left|A_{1}(z)\right|^{2}\left|A_{2}(z)\right|^{2} / 9} \\
k & =\Delta \beta+\frac{1}{3} \gamma\left(P_{1}-P_{2}\right) .
\end{aligned}
$$

Note that in the context of OTDM demultiplexing, the initial idler power is assumed to be zero. The ratio

$$
\eta=\left|A_{4}(z)\right|^{2} /\left|A_{3}(0)\right|^{2}
$$




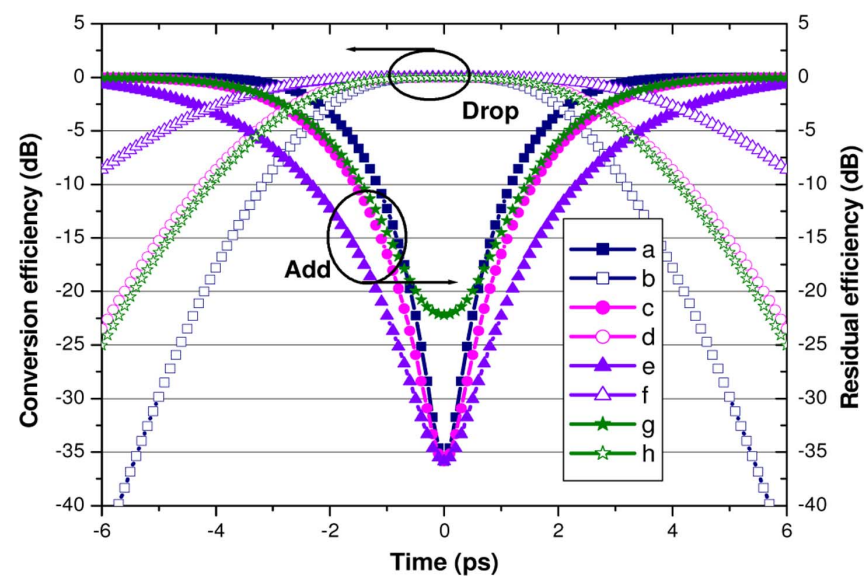

Fig. 2. Theoretical (add, drop) efficiency for various combination of pulse width and phase mismatching conditions [pulse width (ps), phase mismatch $\left.\left(\mathrm{km}^{-1}\right)\right]=(\mathrm{a}, \mathrm{b})[2,0] ;(\mathrm{c}, \mathrm{d})[4,0] ;(\mathrm{e}, \mathrm{f})$ $[6,0] ;(\mathrm{g}, \mathrm{h})[4,0.6]$.

is termed as conversion efficiency in previous literature [1]. Compared with conversion efficiency, we can also denote the residual efficiency such as

$$
\eta_{r}=\left|A_{3}(z)\right|^{2} /\left|A_{3}(0)\right|^{2}=1-\left|A_{4}(z)\right|^{2} /\left|A_{3}(0)\right|^{2}=1-\eta
$$

which arises from the energy conservation and fiber lossless assumption. When one or both pumps are pulsed, the conversion and residual efficiencies are functions of time. Note that, they are now essentially representing the switching window of the drop and add channels, respectively. Since only one pump pulse is used in our experiment, we will confine our theoretical and numerical discussions within the condition that there is only one pulsed pump for the rest of this paper. Let us assume from now on that $A_{1}$ is $\mathrm{CW}$ and $A_{2}$ is pulsed. There is no doubt that this assumption only approximates the switching window because it neglects group velocities mismatch between the pulses and pulse-shape distortion during fiber transmission. However, it is still useful in providing the essential information such as the relationship between pulse width, phase mismatch, and characteristics of switching window.

Curves (a)-(f) in Fig. 2 show the calculated residual (add) and conversion (drop) efficiency when we assume phase mismatch $\Delta \beta=0 \mathrm{~km}^{-1}$, while the fiber parameters used are $\gamma=14 \mathrm{~W}^{-1}$ $\mathrm{km}^{-1}, L=0.4 \mathrm{~km}$. Gaussian-shaped pump pulses with pulse width of 2,4 , and 6 ps and $\left|A_{1}\right|^{2}=\left|A_{2}(t)\right|_{\text {peak }}^{2}=3 \pi / 4 \gamma L=$ $420 \mathrm{~mW}=26.2 \mathrm{dBm}$ are also used according to [16].

It is noticed that the widths of all the drop efficiencies are wide enough to transfer a typical 2-ps wide OTDM signal pulse at $160-\mathrm{Gb} / \mathrm{s}$ to the demultiplexed channel under all three pulse width conditions. Although the full-width at half-maximum (FWHM) of the add efficiencies varied, the add efficiencies at the edges of signal pulses (from -1 to $1 \mathrm{ps}$ ) are still as high as $-15 \mathrm{~dB}$ when the pump pulse width is greater than $4 \mathrm{ps}$. It is already good enough for $160-\mathrm{Gb} / \mathrm{s}$ add performance [21]. To explore the impact of phase matching on add or drop efficiency, a phase mismatch $\Delta \beta \approx 0.6 \mathrm{~km}^{-1}$ is introduced in calculation while the power level and other parameters remain. For 4-ps pump pulse, add and drop efficiencies are shown in curves $(\mathrm{g})$ and (h) in Fig. 2. It can be observed that the drop efficiency does not suffer much while the peak of add efficiency drops by more than $10 \mathrm{~dB}$. In fact, it indicates that the add efficiency of our proposed OTDM demultiplexer is sensitive to phase-matching condition since PWE originates from FWM. The linear phase mismatch $\Delta \beta$ due to chromatic dispersion can be expressed as [1]

$$
\Delta \beta=2 \sum_{m=1}^{\infty} \frac{\beta_{2 m}}{2 m !}\left[\left(\Delta \omega_{4}\right)^{2 m}-\left(\Delta \omega_{3}\right)^{2 m}\right]
$$

In which $\Delta \omega_{3}=\omega_{3}-\omega_{0}, \Delta \omega_{4}=\omega_{4}-\omega_{0}, \beta_{2 m}$ denotes the $2 m$ th derivative of $\beta$ with respect to frequency around the ZDW. To gain better insight into $\Delta \beta$, we limit the summation of (8) to $m=1,2$. According to (8), factors such as fiber dispersion slope, wavelength selection of pumps, and signal will have a critical impact on the add efficiency of the demultiplexer.

Although the previous analytical model demonstrates that PWE is theoretically promising in high-speed applications such as ADM, estimation error can be arisen from neglecting the dispersion and Raman interaction between pumps and signal. It is reported that the walk-off time during which the two pulses interact with each other have an impact on the switching window [17]. Furthermore, it can be predicted that asymmetric power transfer characteristic inherited in WE I will affect the symmetry of switching window. In addition, the variation of pulse shape during fiber transmission due to self-phase modulation (SPM) and the existence of spurious FWM terms are not taken into account. Thus, in order to have a more realistic picture about the switching window, we have performed simulations by using OptSim [22]. We consider two pump waves located at $1540 \mathrm{~nm}$ (CW pump) and 1548 nm (Gaussian-shaped pulsed pump) with the average powers of $25.2 \mathrm{dBm}$ and peak power of $29 \mathrm{dBm}$, respectively. The Gaussian-shaped RZ signals are located at $1561 \mathrm{~nm}$ with the average powers of $-3 \mathrm{dBm}$ and pulse width of $2 \mathrm{ps}$. The fiber parameters used are ZDW $=1554 \mathrm{~nm}, \mathrm{~d} D / \mathrm{d} \lambda=0.03 \mathrm{ps} / \mathrm{nm}^{2}$ $\mathrm{km}, \beta_{4}=10^{-4} \mathrm{ps}^{4} / \mathrm{km}$, and $\gamma=14 \mathrm{~W}^{-1} \mathrm{~km}^{-1}$. These correspond to the experimental parameters as shown in Section III.

First, we measure add and drop windows of our proposed demultiplexer by sliding the Gaussian-shaped control pulse across the signal pulse at $10-\mathrm{Gb} / \mathrm{s}$ base rate and measuring the corresponding output optical powers after filters centered at the wavelengths of the surviving and demultiplexed channel, respectively. Curves (a)-(f) in Fig. 3 correspond to the simulated switching windows when the pulse widths of the pump are 2 , 4, and 6 ps, respectively. It can be observed that the on-off extinction ratios (which is defined as the suppression within the 6 ps time offset from the transmission peak or minimum) of the relative transmission curves for add and drop windows vary significantly with the pulse width. For 4-ps wide pulse, extinction ratios of $\sim 20 \mathrm{~dB}$ are achieved for the drop and add channel and the FWHM of the windows is 3.2 ps and 1.9 ps for the drop and the add channels, respectively. However, for 2-ps wide pulse, the on-off extinction ratio and widths for drop and add channels are $17 \mathrm{~dB}$ and $12 \mathrm{~dB}$ and $2.6 \mathrm{ps}$ and 2.1 ps each. We may also notice that switching windows are not very symmetric especially 


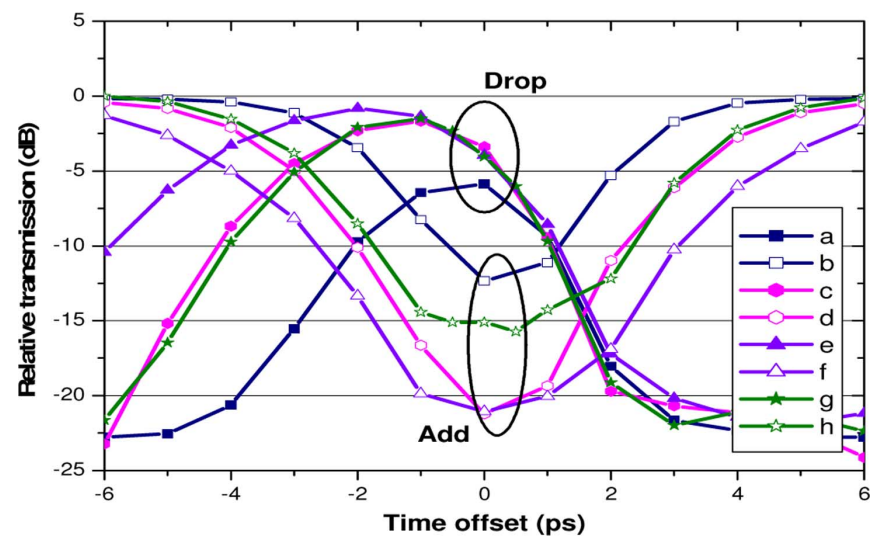

Fig. 3. Simulated switching windows of the demultiplexer for various pulse width and central pulsed-pump wavelength [pulse width (ps), central wavelength $(\mathrm{nm})]=(\mathrm{a}, \mathrm{b})[2,1548] ;(\mathrm{c}, \mathrm{d})[4,1548]$; (e, f) $[6,1548] ;(\mathrm{g}, \mathrm{h})[4,1548.5]$.

for broader pulses. One possible explanation lies in that the unbalanced Raman gain effect for add and drop channel introduce it. On the other hand, it can also be attributed to the pulse-shape distortion suffered by control pulse, which is mainly due to the combined effect of SPM and GVD. Further optimization such as GVD property of the HNL-DSF and reducing the peak pump power should allow for symmetric switching windows [21]. In fact, assuming the dispersion increases linearly with the wavelength around ZDW, the walk-off length $L_{\mathrm{w}}=T_{0} /\left|d_{12}\right|=$ $T_{0} /\left[d D / d \lambda\left(\lambda_{\mathrm{p}}-\lambda_{0}\right)\left(\lambda_{\mathrm{s}}-\lambda_{\mathrm{p}}\right)+d D / d \lambda\left(\lambda_{\mathrm{s}}-\lambda_{\mathrm{p}}\right)^{2}\right] \approx 1 \mathrm{~km}$, where $d_{12}$ is the walk-off parameter and $T_{0}$ is pulse width of the pulsed pump [9], ensures that reducing the pulse peak power by using longer fiber length is a reasonable option.

As we have discussed in curves (g) and (h) in Fig. 2, phase mismatch will largely reduce the residual efficiency according to our analytical model, which corresponds to the switching window for add channel. However, for pulsed-pump-based PWE, the phase-matching condition is not as obvious as CW pump-based PWE. In order to investigate this effect in a more real situation, the wavelength of the pump pulse is detuned from 1548 to $1548.5 \mathrm{~nm}$ on purpose in the OptSim simulation. As shown in curves (g) and (h) in Fig. 3, the on-off extinction ratio for the add channel decreases by $5 \mathrm{~dB}$, while that of the drop channel is not affected.

In Fig. 4(a), this issue is explored further. The central wavelength of the pump pulse is tuned from $1547 \mathrm{~nm}$ to $1549 \mathrm{~nm}$ so as to observe how the on-off extinction ratio varies. The simulation parameters used here are the same as those in Fig. 3 except that the pump pulse width is fixed at 4 ps. We can observe that although the on-off extinction ratio of drop channel is kept at relatively high value $(>15 \mathrm{~dB})$ over the $2 \mathrm{~nm}$ range that of the add channel is high enough $(>18 \mathrm{~dB})$ for a satisfactory add operation only within 0.5 -nm-wide region around 1548 $\mathrm{nm}$. These facts indicate that the wavelength selection for pump pulse is critical for wide-band phase matching, which cannot be revealed from the theoretical calculation shown in Fig. 2.

According to (5), in order to achieve a large on-off extinction ratio for both add and drop channels, the condition that the peak power of pulsed pump equals to $\mathrm{CW}$ pump power must be satisfied if we assume that linear phase mismatch $\Delta \beta \approx 0 \mathrm{~km}^{-1}$.

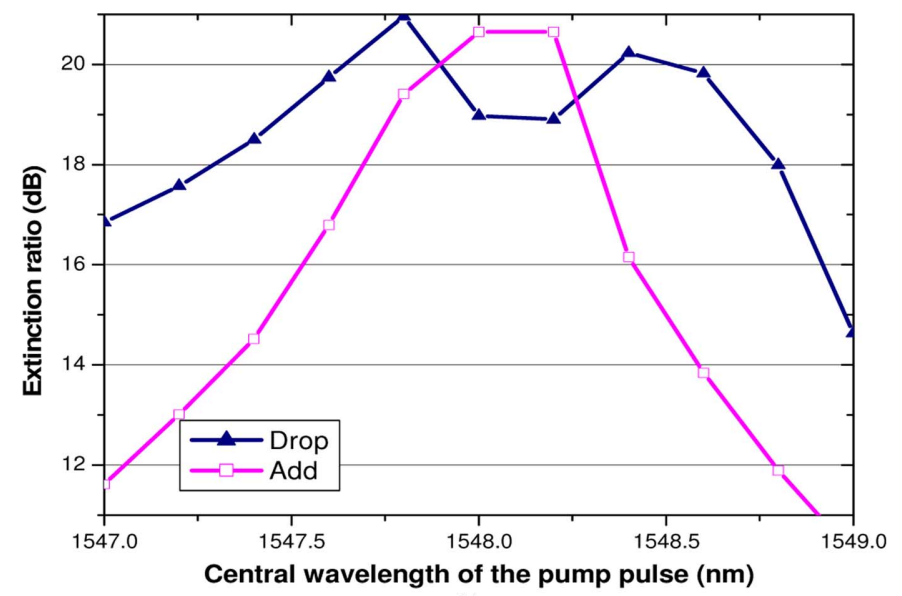

(a)

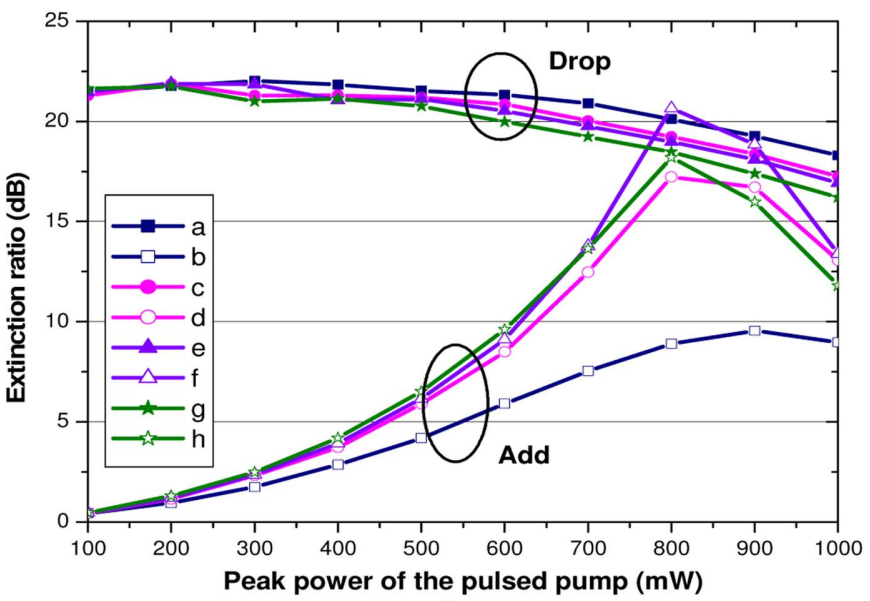

(b)

Fig. 4. (a) Extinction ratios for add and drop channel versus the central wavelength of the pump pulse. (b) Extinction ratios for add and drop channel versus the peak power of pump pulse. The CW pump powers are (a), (b) $200 \mathrm{~mW}$, (c), (d) $300 \mathrm{~mW}$, (e), (f) $330 \mathrm{~mW}$, and (g), (h) $400 \mathrm{~mW}$.

In reality, the power relationship between pulsed pump and $\mathrm{CW}$ pump is not maintained as $\left|A_{1}\right|^{2}=\left|A_{2}(t)\right|_{\text {peak. }}^{2}$. It can be qualitatively attributed to the fact that more than only four frequency components $\left(A_{1}-A_{4}\right)$ are produced and interact with each other in the actual PWE process. In addition, the Raman gain will also exhaust the power of pulsed pump because the frequency of the OTDM signal is exactly $13 \mathrm{THz}$ above that of the pulsed pump. Since much power of the pulsed pump is exhausted during these complicated nonlinear processes, its initial peak power required in experiment is larger than $26.2 \mathrm{dBm}$, which is calculated from $\left|A_{2}(t)\right|_{\text {peak }}^{2}=3 \pi / 4 \gamma L$. However, if the pulsed pump's power level is beyond the value that is required to completely deplete the original OTDM signal, the on-off extinction ratio will decrease due to the periodic nature of PWE. Fig. 4(b) shows the influence of the peak power of the pump pulse and average power of CW pump on the on-off extinction ratio. The parameters used in simulation are the same as in Fig. 3 except that the pump pulse width is fixed at $4 \mathrm{ps}$ and pump powers are varied. It can be observed that an appropriate choice of pump powers is critical to obtain high on-off extinction ratio for both add and drop channels. An optimized combination outcome from simulation is $P_{\mathrm{cW}}=330 \mathrm{~mW}$ and $P_{\text {pulse }}=800 \mathrm{~mW}$. If we compare this set 


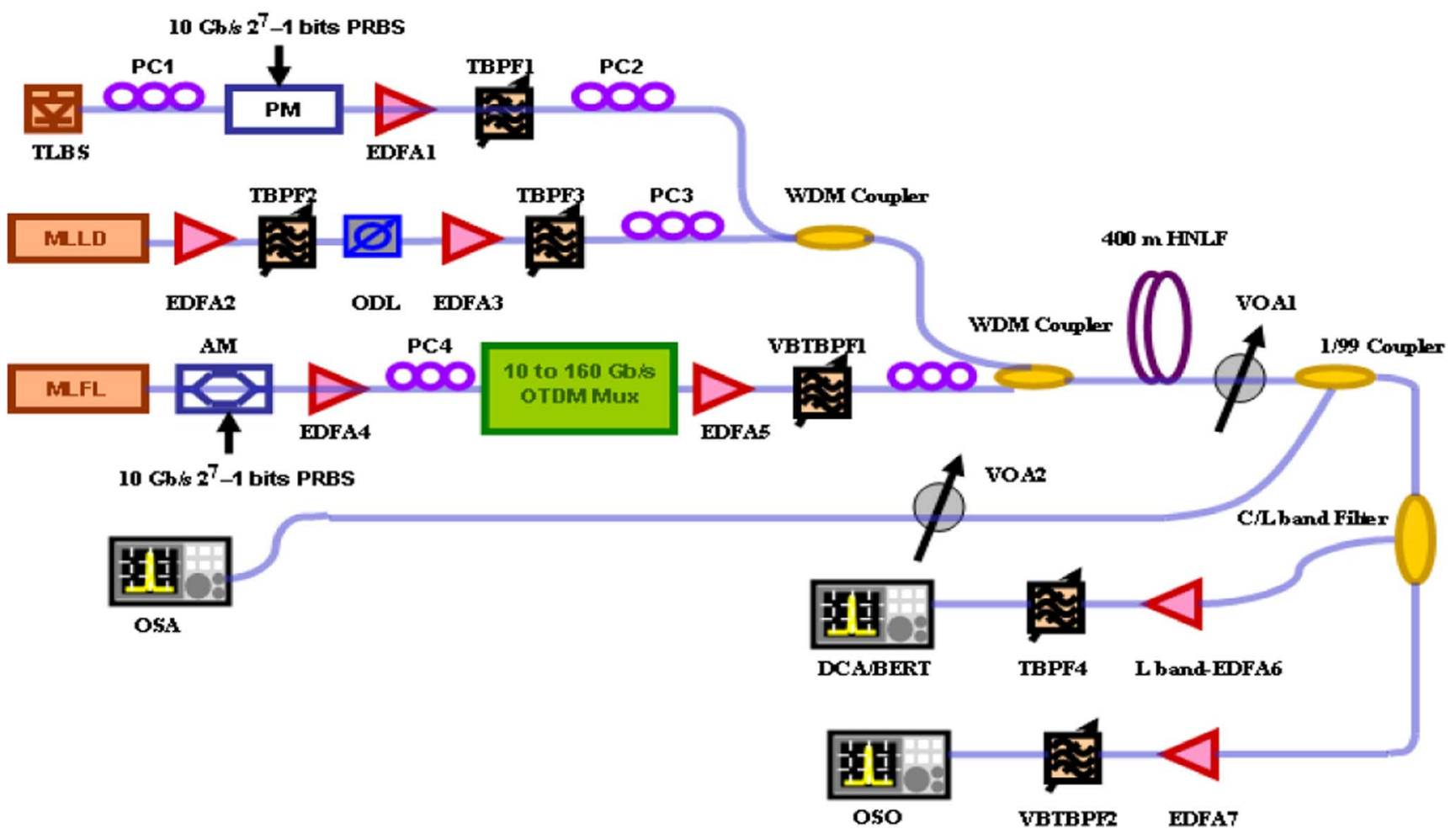

Fig. 5. Experimental setup for the proposed $160 \mathrm{~Gb} / \mathrm{s} \mathrm{demultiplexer.}$

of parameter with the analytical prediction, it can be found that the $\mathrm{CW}$ power is lower but the peak power of the pump pulse is much higher. It matches our qualitative discussion mentioned earlier.

\section{EXPERIMENTAL SETUP}

Fig. 5 shows the experimental setup of the proposed OTDM demultiplexer. The input 160-Gb/s OTDM RZ signal at 1561 $\mathrm{nm}$ is generated by time multiplexing an intensity-modulated 2-ps $10-\mathrm{GHz}$ pulse train from a mode-locked fiber laser with a Mach-Zehnder modulator. An erbium-doped fiber amplifier (EDFA1) is used before the bit-rate multiplier (MUX) to compensate its loss. The pseudorandom binary sequence (PRBS) length of the resultant bit-rate multiplied $160-\mathrm{Gb} / \mathrm{s}$ OTDM signal is $2^{7}-1$ bits. This $160 \mathrm{~Gb} / \mathrm{s}$ signal is then amplified to $8 \mathrm{dBm}$ average power with another stage of EDFA2 before fiber input. To avoid distortion of $160-\mathrm{Gb} / \mathrm{s}$ OTDM signal, the bandwidth of the filter must be broad enough to contain the whole spectra of the signal. Thus, a variable bandwidth tunable bandpass filter (VBTBPF1) is inserted after EDFA2 to reduce amplified spontaneous noise (ASE) level. The bandwidth of the VBTBPF ranges from 0.4 to $10 \mathrm{~nm}$, and its insertion loss is approximately $6 \mathrm{~dB}$. On the other hand, a 10-GHz pump pulse with 2-ps pulse width at $1549 \mathrm{~nm}$ is prepared by a mode-locked laser diode. It is preamplified by EDFA3 before boosted by EDFA4 to a $15-\mathrm{dBm}$ average input power. Two tunable bandpass filters (TBPF1 and TBPF2) are inserted after each EDFA to reduce ASE noise as well. The optical delay line placed in the middle of two amplifier sections is used to align the gating pulse with the bit slot of the target channel. The CW pump at $1540 \mathrm{~nm}$ is phase dithered with a phase modulator driven by a 10-Gb/s $2^{7}-1$ PRBS to suppress stimulated Brillouin scattering [23]. The CW pump is amplified by EDFA5 to 26- $\mathrm{dBm}$ average input power and combined with the pulsed pump. The state of polarization of the pumps and signals are controlled by polarization controller (PC2-4) at each arm to satisfy the orthogonal polarization condition before they are launched into a 400-m HNL-DSF for signal demultiplexing. The optical spectra before and after HNL-DSF are shown in Fig. 6(a). The nonlinear coefficient $\gamma$, the ZDW $\lambda_{0}$, and the dispersion slope $d D / d \lambda$ of the HNL-DSF are $14 \mathrm{~W}^{-1} \mathrm{~km}^{-1}, 1554 \mathrm{~nm}$, and $0.03 \mathrm{ps} / \mathrm{nm}^{2} \mathrm{~km}$, respectively. After demultiplexing, the surviving $150 \mathrm{~Gb} / \mathrm{s}$ signal and demultiplexed $10 \mathrm{~Gb} / \mathrm{s}$ signals are separately filtered and amplified by TBPF4 and VTBPF2 and corresponding EDFAs before being sent to digital communication analyzer (DCA) and optical sampling oscilloscope (OSO) for eye diagrams measurement, respectively. The OSO working in C-band has a very wide bandwidth $>500 \mathrm{GHz}$ and short sampling resolution $\approx 1 \mathrm{ps}$.

\section{EXPERIMENTAL RESULTS AND DISCUSSION}

In order to characterize the proposed demultiplexer, Fig. 6(b) shows the measured add and drop windows. We use the same method as we discussed in Section II to measure them, which is to time shift the control pump pulse across the $10-\mathrm{Gb} / \mathrm{s}$ RZ signal pulse while measuring the output powers at the wavelength of signal and demultiplexed channel, respectively. Although we do not realize the add operation in experiment, the switching window for the add channel is still measured to demonstrate the potential to upgrade our proposed demultiplexer to an ADM. The FWHM of the measured windows is 6.3 ps and $2.0 \mathrm{ps}$ for the drop and the add channels, respectively. 


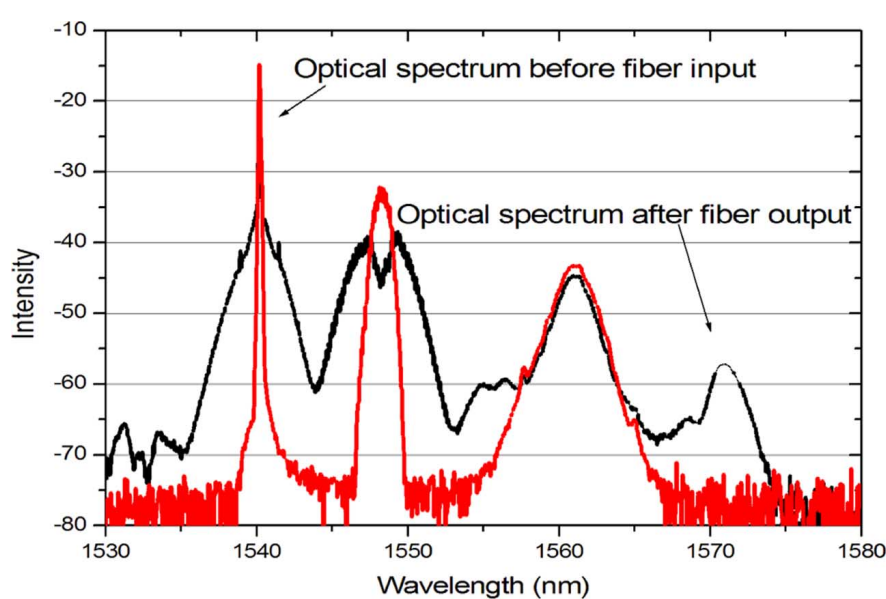

(a)

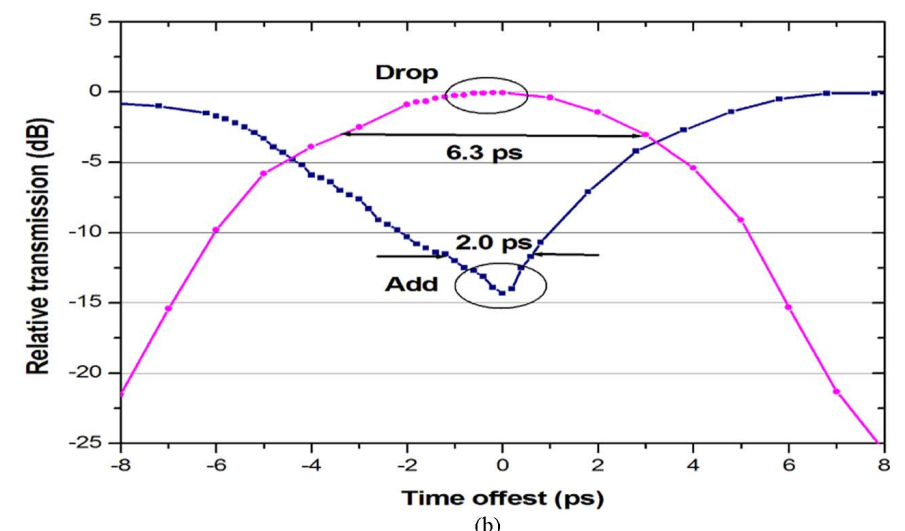

Fig. 6. (a) Optical spectra before and after HNLF. (b) Measured switching windows for add and drop channels.

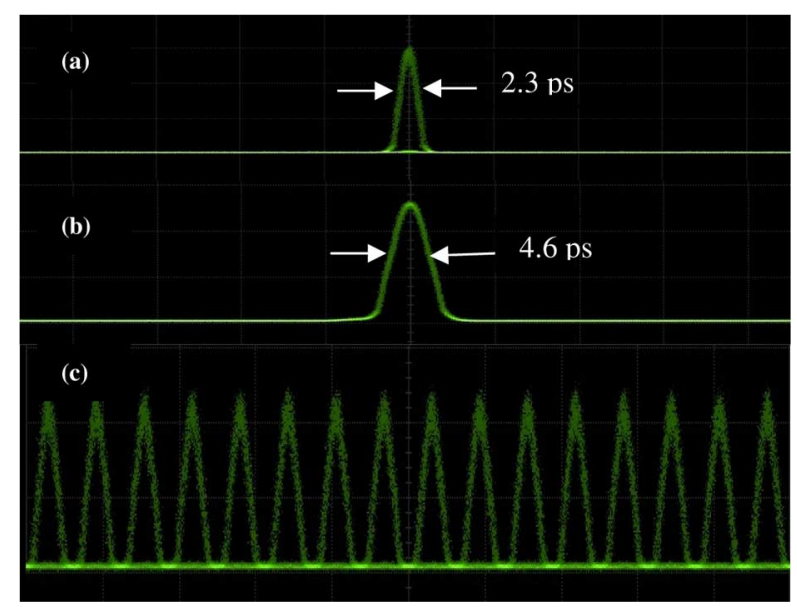

Fig. 7. Measured eye diagrams by OSO. (a) Original 10- Gb/s RZ signal. (b) Pump pulse before fiber input. (c) $160-\mathrm{Gb} / \mathrm{s}$ multiplexed data. Time base: $10 \mathrm{ps} / \mathrm{div}$.

The corresponding on-off extinction ratios are $\sim 22 \mathrm{~dB}$ and $\sim 15 \mathrm{~dB}$, which match qualitatively with the simulated results shown in the curves $(\mathrm{g})$ and $(\mathrm{h})$ in Fig. 3.

Fig. 7(a) shows the 10-GHz RZ signal pulse before the MUX. Its pulse width is measured to be 2.3 ps. Fig. 7(b) shows the $10-\mathrm{GHz}$ pump pulse. Its pulse width is broadened to $4.6 \mathrm{ps}$ by

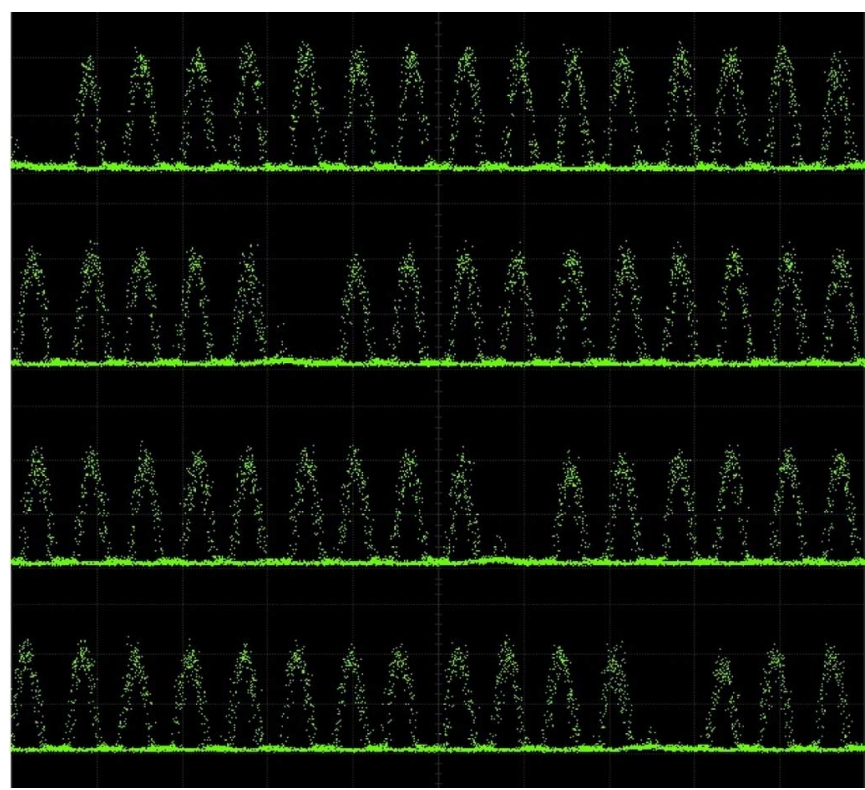

Fig. 8. Measured eye diagrams for the surviving channels by OSO. From top to bottom, channels \#1, \#6, \#10, and \#13. Time base: $10 \mathrm{ps} / \mathrm{div}$.

the two 0.8-nm tunable filters TBPF1 and TBPF2. The 160-Gb/s multiplexed OTDM signal is shown in Fig. 7(c) for reference.

To demonstrate the potential simultaneous add/drop operations at a $160-\mathrm{Gb} / \mathrm{s}$ signal data rate, eye diagrams for some of 16 surviving channels and after demultiplexer are shown in Fig. 8. Two odd and two even channels are randomly selected and displayed. They are channels 1, 6, 10, and 13 from top to bottom, respectively. It can be observed that the target channel is almost completely depleted in all cases and clear and widely open eye diagrams are observed for the surviving signals. The small amount of residual power at the original channel can be attributed to combined effects of incomplete exchange and preamplification before OSO. The incomplete exchange may arise from the ZDW fluctuation within the fiber and is reflected by the finite on-off extinction ratio of the add window. A disadvantage of the PWE-based demultiplexer compared to the popular NOLM-based demultiplexer is that the latter one has no phase-matching requirement, and hence, $\mathrm{ZDW}$ variations do not influence the performance of the system. One thing noteworthy is that preamplification is necessary because the sensitivity of the OSO is relatively poorer than conventional photodetector. Although the amplification raises the amplitude of the surviving channels, it introduces noise as well.

Fig. 9(a) shows the waveforms of the corresponding demultiplexed signals. They are measured by using DCA because its L-band wavelength is beyond the operating range of OSO (C-band). The tail's ripples observed in the demultiplexed signals are due to the limited electrical bandwidth of a $53-\mathrm{GHz}$ photodetector. To investigate the signal quality degradation after demultiplexing, magnified demultiplexed and original signals are compared in Fig. 9(b) and (c). It is noticed that the mark level of the demultiplexed signal is slightly noisier compared with original one. It is believed to be caused by the phase dithering of the CW pump light. The CW pump's dithered phases are transferred to the idler through PWE 


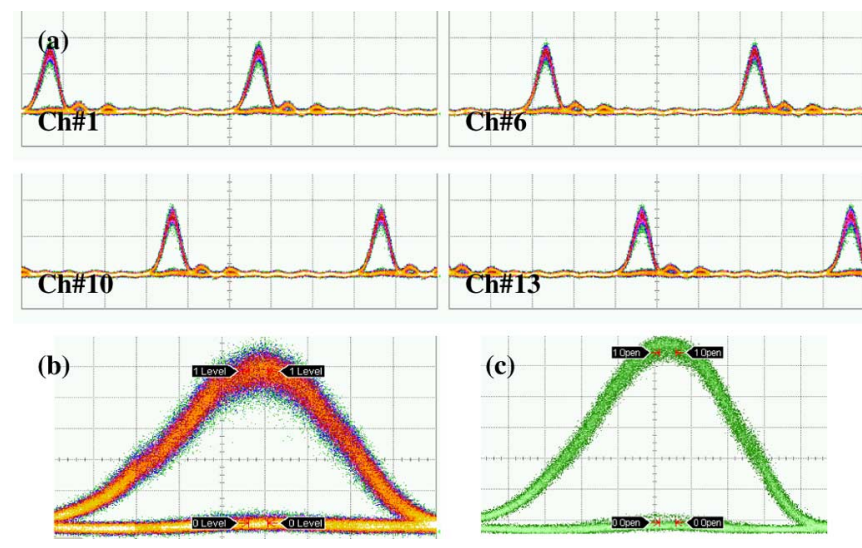

Fig. 9. (a) Measured eye diagrams for the four demultiplexed channels. Time base: $10 \mathrm{ps} / \mathrm{div}$. (b) Magnified eye diagrams for the demultiplexed signal. (c) Magnified eye diagrams for the original signal.

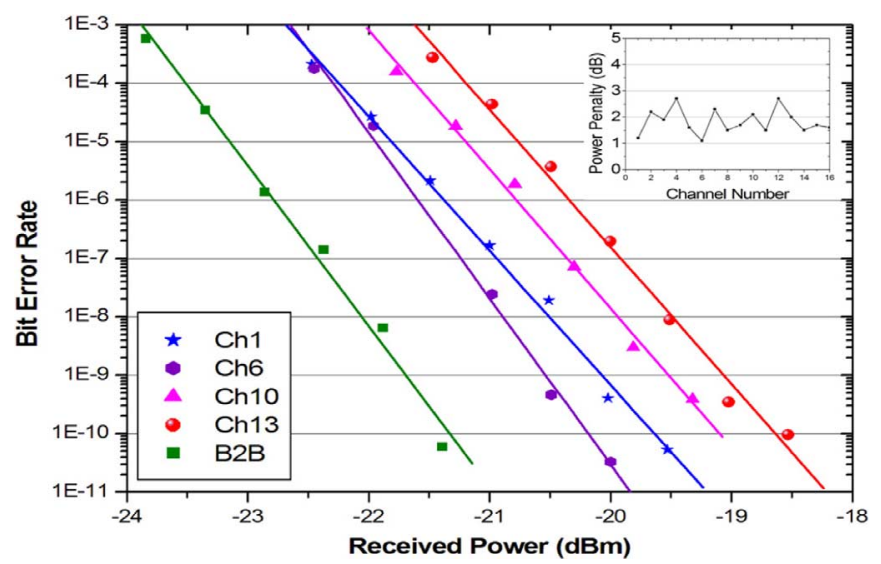

Fig. 10. BER plots of the B2B and four demultiplexed signals (inset). Power penalty at $10^{-9}$ for all 16 channels.

process. Furthermore, the phase modulation is converted to intensity modulation during fiber transmission and filter. Such degradation can be avoided by using two pulsed pumps.

To quantify the performance of the demultiplexer, bit-error rate (BER) of the demultiplexed signals are measured and compared against the back-to-back (B2B) 10-Gb/s RZ signal as shown in Fig. 10. Error-free operations are attained for all channels. Compared to the B2B curve, the power penalties incurred in the demultiplexed process range from 1.2 to $2.7 \mathrm{~dB}$ at $10^{-9}$ BER as shown in the inset of Fig. 10. The variation of power penalty among different channels is believed to be caused by amplitude fluctuation of the bit-rate multiplied OTDM signal.

\section{CONCLUSION}

We have theoretically investigated the switching windows of our proposed OTDM demultiplexer based on PWE in HNLF, which is shown to be a promising candidate in high-speed signal processing application. It is also verified by the simulation results, which take chromatic dispersion and other nonlinear effects such as Raman effect into account. The investigation toward the dependence of on-off extinction ratio of switching window on the phase-mismatch condition and pump power levels reveals the optimized parameter selection for the proposed demultiplexer. We also have experimentally demonstrated the OTDM demultiplexer. One 160-Gb/s OTDM signal has been successfully demultiplexed into 16 individual $10-\mathrm{Gb} / \mathrm{s}$ signals. Clear and widely open eye diagrams have been obtained for all 16 demultiplexed channels, with less than or equal to $2.7 \mathrm{~dB}$ power penalty at BER of $10^{-9}$. The clean depletion performance at the target time slot for surviving channels demonstrates that the proposed demultiplexer has potential capability for ADM of high-speed OTDM signals. The polarization insensitive operation of wavelength exchange using polarization diversity scheme [24] or potentially circularly polarized pumps [25] can be advantageous for its deployment compared to its NOLM counterpart [26]. Experimental implementation of PWE-based ADM holding these strengths will be the subject of further research.

\section{ACKNOWLEDGMENT}

The authors thank Sumitomo Electric Industries for providing the HNLF and Alnair Laboratories for providing the VBTBPF and OSO.

\section{REFERENCES}

[1] M. E. Marhic, Y. Parker, F. S. Yang, and L. G. Kazovsky, "Widely tunable spectrum translation and wavelength exchange by four-wave mixing in optical fibers," Opt. Lett., vol. 21, no. 23, pp. 1906-1908, 1996.

[2] K. Uesaka, K. K. Y. Wong, M. E. Marhic, and L. G. Kazovsky, "Wavelength exchange in a highly nonlinear dispersion-shifted fiber: Theory and experiments," IEEE J. Sel. Topics Quantum Electron., vol. 8, no. 3, pp. 560-568, May/Jun. 2002.

[3] R. W. L. Fung, H. K. Y. Cheung, P. P. Kuo, C. H. Kwok, and K. K. Y. Wong, "Wavelength exchange in the anomalous-dispersion regime," in Proc. Eur. Conf. Opt. Commun. (ECOC), Cannes, France, 2006, Paper Th 1.3. 2.

[4] R. W. L. Fung, H. K. Y. Cheung, and K. K. Y. Wong, "Widely tunable wavelength exchange in anomalous-dispersion regime," Photon. Technol. Lett., vol. 19, no. 22, pp. 1846-1848, 2007.

[5] M. Z. Shen, R. W. L. Fung, H. K. Y. Cheung, and K. K. Y. Wong, "A comprehensive study on the dynamic range of wavelength exchange and its impact on exchanged signal performance," J. Lightw. Technol., vol. 27, pp. 2707-2716, 2009.

[6] K. K. Y. Wong, M. E. Marhic, K. Uesaka, and L. G. Kazovsky, "Wavelegnth exchange: A novel function for optical networks," Inf. Sci., no. 149, pp. 161-169, 2003

[7] H. K. Y. Cheung, R. W. L. Fung, C. H. Kwok, and K. K. Y. Wong, "All-optical packet switching by pulsed-pump wavelength exchange in a highly nonlinear dispersion-shifted fiber," in Proc. OFC, 2007, Paper OTuB4.

[8] K. K. Y. Wong, H. K. Y. Cheung, R. W. L. Fung, C. H. Kwok, B. K. K. Kuo, and P. C. Chui, "Versatile parametric wavelength exchange," in Proc. LEOS Winter Topicals, Invited Paper, Innsbruck, Austria, 2009.

[9] G. P. Agrawal, Nonlinear Fiber Optics, 3rd ed. San Diego, CA: Academic, 2001, ch.8.

[10] T. Ohara, H. Takara, I. Shake, K. Mori, K. Sato, S. Kawanishi, S. Mino, T. Yamada, M. Ishii, I. Ogawa, T. Kitoh, K. Magari, M. Okamoto, R. V. Roussev, J. R. Kurz, K. R. Parameswarn, and M. M. Fejer, "160-Gb/s OTDM transmission using integrated all-optical MUX/DEMUX with all-channel modulation and demultiplexing," IEEE Photon. Technol. Lett., vol. 16, pp. 650-652, Feb. 2004.

[11] U. Feiste, R. Ludwig, C. Schubert, J. Berger, C. Schmidt, J. Berger, C. Schmidt, H. G. Weber, B. Schmauss, A. Munk, B. Buchold, D. Briggmann, F. Kueppers, and F. Rumpf, " $160 \mathrm{Gbit} / \mathrm{s}$ transmission over 116 $\mathrm{km}$ field-installed fibre using $160 \mathrm{Gbit} / \mathrm{s}$ OTDM and $40 \mathrm{Gbit} / \mathrm{s}$ ETDM," Electron. Lett., vol. 37, pp. 443-445, 2001

[12] H. S. Chung, S. K. Shin, D. W. Lee, D. W. Kim, and Y. C. Chung, "640 Gbit/s $(32 \times 20 \mathrm{Gbit} / \mathrm{s})$ WDM transmission with $0.4(\mathrm{bit} / \mathrm{s}) / \mathrm{Hz}$ spectral efficiency using short-period dispersion-managedfibre," Electron. Lett., vol. 37, pp. 618-620, 2001.

[13] T. Morioka, H. Takara, S. Kawanishi, T. Kitoh, and M. Saruwatari, "Error-free $500 \mathrm{Gbit} / \mathrm{s}$ all-optical demultiplexing using low-noise, low-jitter supercontinuum short pulses," Electron. Lett., vol. 32, pp. 833-834, 1996 
[14] T. Yamamoto, E. Yoshida, and M. Nakazawa, "Ultrafast nonlinear optical loop mirror for demultiplexing $640 \mathrm{Gbit} / \mathrm{s}$ TDM signals," Electron. Lett., vol. 34, pp. 1013-1014, 1998.

[15] J. Glesk, J. P. Sokoloff, and P. R. Prucnal, "Demonstration of all-optical demultiplexing of TDM data at $250 \mathrm{Gbit} / \mathrm{s}$," Electron. Lett., vol. 30, pp. 339-341, 1994.

[16] C. H. Kwok, B. P. P. Kuo, and K. K. Y. Wong, "Pulsed pump wavelength exchange for high speed signal de-multiplexing," Opt. Exp., vol. 16, pp. 10894-10899, 2008.

[17] P. O. Hedekvist, M. Karlsson, and P. A. Andrekson, "Fiber four-wave mixing demultiplexing with inherent parametric amplification," $J$. Lightw. Technol., vol. 15, pp. 2051-2058, Nov. 1997.

[18] Y. J. Chen, "Four-wave mixing in optical fibers: Exact solution," J. Opt. Soc. Am. B, vol. 6, no. 11, pp. 1986-1993, 1989.

[19] G. Cappellini and S. Trillo, "Third-order three-wave mixing in singlemode fibers: Exact solutions and spatial instability effects," J. Opt. Soc. Am. B, vol. 8, no. 4, pp. 824-838, 1991.

[20] T. Torounidis, M. Karlsson, and P. Andrekson, "Fiber optical parametric amplifier pulse source: Theory and experiments," J. Lightw. Technol., vol. 23, pp. 4067-4073, Dec. 2005.

[21] J. H. Lee and K. Kikuchi, "All-fiber-based 160-Gbit/s add/drop multiplexer incorporating a 1-m-long bismuth oxide-based ultra-high nonlinearity fiber," Opt. Exp., vol. 13, pp. 6864-6869, 2005.

[22] OptSim, Distributed by RSoft Feb. 2004 [Online]. Available: http:// www.rsoftdesign.com

[23] S. K. Korotky, P. B. Hansen, L. Eskildsen, and J. J. Veselka, "Efficient phase modulation scheme for suppression of stimulated brillouin scattering," IOOC 1995, vol. WD2-1, pp. 109-111, 1995.

[24] K. Uesaka, K. K. Y. Wong, M. E. Marhic, and L. G. Kazovsky, "Polarization-insensitive wavelength exchange in highly-nonlinear dispersion shifted fiber," in Proc. OFC, 2002, Paper ThY3.

[25] M. E. Marhic, K. K. Y. Wong, and L. G. Kazovsky, "Fiber optical parametric amplifiers with linearly or circularly polarized waves," $J$. Opt. Soc. Am. B, vol. 20, no. 12, pp. 2425-2433, 2003.

[26] J. H. Lee, T. Tanemura, Y. Takushima, and K. Kikuchi, "All-optical 80-Gb/s add-drop multiplexer using fiber-based nonlinear optical loop mirror," IEEE Photon. Technol. Lett., vol. 17, pp. 840-842, Apr. 2005.

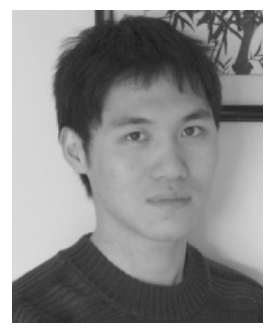

Mengzhe Shen received the B.S. degree in physics from the Shanghai Jiaotong University, Shanghai, China, in 2007. He is currently working toward the M.Phil. degree in electronic engineering at The University of Hong Kong, Hong Kong.

His current research interests include applications of fiber optical parametric effect and fiber nonlinearity in optical communication systems.

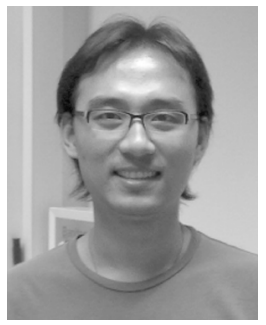

Xing Xu received the B.Eng. degree in telecommunication engineering from Xidian University, Xi' an, China, in 2004. Currently, he is working toward the M.Phil. degree in electronic engineering at The University of Hong Kong, Hong Kong.

From 2004 to 2007, he worked as an R\&D engineer with Fiberhome Telecommunication Co. Ltd. During 2007 and 2008, he was a Graduate Student at Delft University of Technology, Delft, The Netherlands.

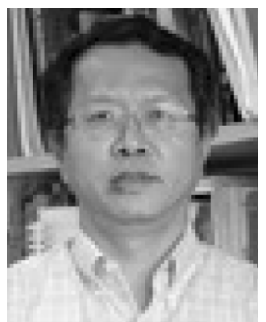

T. I. Yuk received the B.S. degree from Iowa State University, Ames, IA, in 1978, and the M.S. and $\mathrm{Ph} . \mathrm{D}$. degrees from Arizona State University, Tempe, AZ, in 1980 and 1986, respectively.

Since 1986, he has been a Lecturer at the University of Hong Kong (HKU), Hong Kong. His current research interests include modeling of mode locking techniques in fiber soliton laser, time-domain modeling of waveguide laser, bandwidth management and congestion control in broadband wide-area and local-area networks, protocol design for lightwave,

and wireless networks.

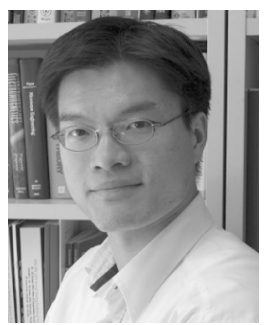

Kenneth Kin-Yip Wong (S'00-M'03) received combined B.E. (first class honors with medal award) degree in electrical engineering and B.S. degree in physics from the University of Queensland, Brisbane, Australia, in 1997, and the M.S. and Ph.D. degrees, both in electrical engineering, in 1998 and 2003, respectively, from Stanford University, Stanford, CA.

He worked in Hewlett-Packard Laboratories as a Research Engineer and contributed in projects included parallel optics and VCSEL in 1998-1999. $\mathrm{He}$ also worked as an independent consultant in Innovation CORE (A Sumitomo Electric Company), CA, in 2004. He was a member of the Photonics and Networking Research Laboratory, Stanford University. Currently, he is an Assistant Professor in the Department of Electrical and Electronic Engineering, The University of Hong Kong, Hong Kong. His research interests include DWDM systems, SCM optical systems, fiber nonlinearity, fiber optical parametric amplifiers, and photonic crystal fibers. $\mathrm{He}$ has authored or coauthored more than 100 journal and conference papers.

Dr. Wong was the recipient of OSA New Focus Student Award and IEEE/ LEOS Graduate Student Fellowship, both in 2003. He is the reviewer for Optics Letters, JOSA B, Optics Express, IEEE PHOTONICS TECHNOLOGY LETTERS, IEEE/OSA JOURNAL OF LIGHTWAVE TECHNOLOGY, and IEEE LETTERS AND OPTICS COMMUNICATIONS. He won the Best Teacher Award in 2005-2006. He is a member of the OSA, IEEE, and IEEE Photonics Society. 